\title{
Fractional Derivatives of Some Fractional Functions and Their Applications
}

\author{
Chii-Huei Yu \\ Associate Professor, School of Mathematics and Statistics, Zhaoqing University, Guangdong Province, China. Email: chiihuei@139.com
}

Article Received: 09 January $2020 \quad$ Article Accepted: 27 February $2020 \quad$ Article Published: 24 March 2020

\section{ABSTRACT}

In this paper, we make use of the fractional differential operator method to find the modified Riemann-Liouville (R-L) fractional derivatives of some fractional functions include fractional polynomial function, fractional exponential function, fractional sine and cosine functions. The Mittag-Leffler function plays an important role in our article, and the fractional differential operator method can be applied to find the particular solutions of non-homogeneous linear fractional differential equations (FDE) with constant coefficients in a unified way and it is a generalization of the method of finding particular solutions of classical ordinary differential equations. On the other hand, several examples are illustrative for demonstrating the advantage of our approach and we compare our results with the traditional differential calculus cases.

Keywords: Fractional differential operator method, Modified R-L fractional derivatives, Fractional functions, Mittag-Leffler function, Particular solutions, Non-homogeneous linear FDE with constant coefficients.

\section{INTRODUCTION}

Fractional Calculus arises from a question proposed by L'Hospital and Lebniz in 1695, it is the generalization of traditional integer-order calculus. Reviewing the history, the fractional calculus was more interesting topic to mathematicians for a long time in spite of the lack of application background. Upcoming years more and more researchers have paid their attention towards fractional calculus which are used in real world problems such as applied mathematics, physics, mathematical biology and engineering, etc [1-7]. With the great efforts of researchers there have been rapid developments on the theory of fractional calculus and its applications. But the rule of fractional derivative is not unique, the definition of fractional derivative is given by many authors. The commonly used definition is the Riemann-Liouvellie (R-L) fractional derivative [1-2]. Other useful definition includes Caputo definition of fractional derivative (1967) [1], the Grunwald-Letinikov (G-L) fractional derivative [1], and Jumarie's modified R-L fractional derivative is used to avoid nonzero fractional derivative of a constant functions [8]. In [9], Ghosh et al developed analytical method for solution of linear fractional differential equations with Jumarie type of modified R-L derivative. The differential equations in different form of fractional derivatives give different type of solutions.

Therefore, there is no standard methods to solve fractional differential equations (FDE). In this article, we use the fractional differential operator method to obtain the fractional derivatives of some fractional functions, for example, fractional polynomial function, fractional exponential function, fractional sine and cosine function. Moreover, these results can be applied to find the particular solution of non-homogeneous linear FDE with constant coefficients in a unified form which is different from [10-11], and it is a generalization of the method proposed by [13]. The Mittag-Leffler function plays an important role in this study, and the results obtained by traditional differential calculus are special cases of ours. On the other hand, we give some examples to demonstrate the new results and classical ones.

\section{PRELIMINARIES}

In this section, we introduce some fractional functions and their properties. In addition, fractional Euler's formula and DeMoivre's formula are proved. 
Notation 2.1: If $\alpha$ is a real number, then $[\alpha]=\left\{\begin{array}{c}0 \quad \text { if } \alpha<0, \\ \text { the greatest integer less than or equal to } \alpha \text { if } \alpha \geq 0 .\end{array}\right.$

Definition 2.2: Let $\alpha$ be a real number, $m$ be a positive integer, and $f(x) \in C^{[\alpha]}([a, b])$. The modified Riemann-Liouville fractional derivatives of Jumarie type ([8]) is defined by

$$
{ }_{a} D_{x}^{\alpha}[f(x)]=\left\{\begin{array}{cc}
\frac{1}{\Gamma(-\alpha)} \int_{a}^{x}(x-\tau)^{-\alpha-1} f(\tau) d \tau, & \text { if } \alpha<0 \\
\frac{1}{\Gamma(1-\alpha)} \frac{d}{d x} \int_{a}^{x}(x-\tau)^{-\alpha}[f(\tau)-f(a)] d \tau, & \text { if } 0 \leq \alpha<1 \\
\left({ }_{a} D_{x}^{\alpha-m}\right)^{m}[f(x)], & \text { if } m \leq \alpha<m+1
\end{array}\right.
$$

where $\Gamma(y)=\int_{0}^{\infty} t^{y-1} e^{-t} d t$ is the gamma function defined on $y>0$, and $\left({ }_{a} D_{x}^{\alpha}\right)^{n}=\left({ }_{a} D_{x}^{\alpha}\right)\left({ }_{a} D_{x}^{\alpha}\right) \cdots\left({ }_{a} D_{x}^{\alpha}\right)$ is the $n$ times fractional derivative with respect to ${ }_{a} D_{x}^{\alpha}$. We note that $\left({ }_{a} D_{x}^{\alpha}\right)^{n} \neq{ }_{a} D_{x}^{n \alpha}$ in general, and we have the following property [9].

Proposition 2.3: Let $\alpha, \beta, c$ be real numbers and $\beta \geq \alpha>0$, then

$$
{ }_{0} D_{x}^{\alpha}\left[x^{\beta}\right]=\frac{\Gamma(\beta+1)}{\Gamma(\beta-\alpha+1)} x^{\beta-\alpha},
$$

and

$$
{ }_{0} D_{x}^{\alpha}[c]=0 .
$$

Definition 2.4 ([12]): The Mittag-Leffler function is defined by

$$
E_{\alpha}(z)=\sum_{k=0}^{\infty} \frac{z^{k}}{\Gamma(k \alpha+1)}
$$

where $\alpha$ is a real number, $\alpha \geq 0$, and $z$ is a complex number.

Some interesting special cases of Mittag-Leffler function are as listed below:

$$
\begin{aligned}
& E_{0}(z)=\frac{1}{1-z}, \text { if }|z|<1 \\
& E_{1}(z)=e^{z}, \\
& E_{2}(z)=\cosh (\sqrt{z}) .
\end{aligned}
$$

The Mittag-Leffler function has gained importance and popularity during the last one and a half decades due mainly to its applications in the solution of fractional-order differential, integral and difference equations arising in certain problems of mathematical, physical, biological and engineering sciences.

Definition 2.5: Let $0<\alpha \leq 1, \lambda$ be a complex number, $n$ be a non-negative integer, $c_{0}, c_{1}, \cdots, c_{n}$ be real constants, $c_{n} \neq 0$, and $x$ be a real variable. Then

$$
p\left(x^{\alpha}\right)=\sum_{k=0}^{n} c_{k} p_{k}\left(x^{\alpha}\right)
$$

is called $\alpha$-order fractional polynomial function of degree $n$, where $p_{k}\left(x^{\alpha}\right)=\frac{1}{\Gamma(k \alpha+1)} x^{k \alpha}$ for all $k$. 
And $E_{\alpha}\left(\lambda x^{\alpha}\right)$ is called $\alpha$-order fractional exponential function.

Definition 2.6 ([9]): The $\alpha$-order fractional cosine and sine function are defined as follows:

$$
\cos _{\alpha}\left(x^{\alpha}\right)=\sum_{k=0}^{\infty} \frac{(-1)^{k} x^{2 k \alpha}}{\Gamma(2 k \alpha+1)}
$$

and

$$
\sin _{\alpha}\left(x^{\alpha}\right)=\sum_{k=0}^{\infty} \frac{(-1)^{k} x^{(2 k+1) \alpha}}{\Gamma((2 k+1) \alpha+1)}
$$

where $0<\alpha \leq 1$ and $x$ is a real variable.

Remark 2.7: If $\alpha=1$, then $\cos _{1}(x)=\cos x$, and $\sin _{1}(x)=\sin x$.

Notation 2.8: Let $z=a+i b$ be a complex number, where $i=\sqrt{-1}$, and $a, b$ are real numbers. $a$, the real part of $z$, is denoted by $\operatorname{Re}(z) ; b$, the imaginary part of $z$, is denoted by $\operatorname{Im}(z)$.

Proposition 2.9 (fractional Euler's formula): Let $0<\alpha \leq 1$, then

$$
E_{\alpha}\left(i x^{\alpha}\right)=\cos _{\alpha}\left(x^{\alpha}\right)+i \sin _{\alpha}\left(x^{\alpha}\right) .
$$

Proof $E_{\alpha}\left(i x^{\alpha}\right)=\operatorname{Re}\left[E_{\alpha}\left(i x^{\alpha}\right)\right]+i \operatorname{Im}\left[E_{\alpha}\left(i x^{\alpha}\right)\right]$

$$
\begin{aligned}
& =\frac{1}{2}\left[E_{\alpha}\left(i x^{\alpha}\right)+E_{\alpha}\left(-i x^{\alpha}\right)\right]+i \frac{1}{2 i}\left[E_{\alpha}\left(i x^{\alpha}\right)-E_{\alpha}\left(-i x^{\alpha}\right)\right] \\
& =\frac{1}{2}\left[\sum_{k=0}^{\infty} \frac{i^{k} x^{k \alpha}}{\Gamma(k \alpha+1)}+\sum_{k=0}^{\infty} \frac{(-1)^{k} i^{k} x^{k \alpha}}{\Gamma(k \alpha+1)}\right]+i \frac{1}{2 i}\left[\sum_{k=0}^{\infty} \frac{i^{k} x^{k \alpha}}{\Gamma(k \alpha+1)}-\sum_{k=0}^{\infty} \frac{(-1)^{k} i^{k} x^{k \alpha}}{\Gamma(k \alpha+1)}\right] \\
& =\left[\sum_{k=0}^{\infty} \frac{(-1)^{k} x^{2 k \alpha}}{\Gamma(2 k \alpha+1)}\right]+i\left[\sum_{k=0}^{\infty} \frac{(-1)^{k} x^{(2 k+1) \alpha}}{\Gamma((2 k+1) \alpha+1)}\right] \\
& =\cos _{\alpha}\left(x^{\alpha}\right)+i \sin _{\alpha}\left(x^{\alpha}\right) .
\end{aligned}
$$

Remark 2.10: If $\alpha=1$, we obtain Euler's formula $e^{i x}=\cos x+i \sin x$.

Proposition 2.11 (fractional DeMoivre's formula): Let $0<\alpha \leq 1$, and $n$ be a positive integer, then

$$
\left[\cos _{\alpha}\left(x^{\alpha}\right)+i \sin _{\alpha}\left(x^{\alpha}\right)\right]^{n}=\cos _{\alpha}\left(n x^{\alpha}\right)+i \sin _{\alpha}\left(n x^{\alpha}\right) .
$$

Proof By [9], we have

$$
E_{\alpha}\left(\lambda x^{\alpha}\right) \cdot E_{\alpha}\left(\mu x^{\alpha}\right)=E_{\alpha}\left((\lambda+\mu) x^{\alpha}\right),
$$

for any complex numbers $\lambda, \mu$. Therefore,

$$
E_{\alpha}\left(\lambda_{1} x^{\alpha}\right) \cdots E_{\alpha}\left(\lambda_{n} x^{\alpha}\right)=E_{\alpha}\left(\left(\lambda_{1}+\cdots+\lambda_{n}\right) x^{\alpha}\right),
$$

for any complex numbers $\lambda_{1}, \cdots, \lambda_{n}$.

Let $\lambda_{1}=\cdots=\lambda_{n}=i$, we have 


$$
\left[E_{\alpha}\left(i x^{\alpha}\right)\right]^{n}=E_{\alpha}\left(i n x^{\alpha}\right) .
$$

Thus,

$$
\left[\cos _{\alpha}\left(x^{\alpha}\right)+i \sin _{\alpha}\left(x^{\alpha}\right)\right]^{n}=\left[E_{\alpha}\left(i x^{\alpha}\right)\right]^{n}=E_{\alpha}\left(i n x^{\alpha}\right)=\cos _{\alpha}\left(n x^{\alpha}\right)+i \sin _{\alpha}\left(n x^{\alpha}\right)
$$

q.e.d.

Remark 2.12: Let $\alpha=1$, we get the classical DeMoivre's formula $(\cos x+i \sin x)^{n}=\cos n x+i \operatorname{sinn} x$.

\section{METHODS AND RESULTS}

In the following, we give a definition of infinite fractional differentiable function.

Definition 3.1: Assume that $0<\alpha \leq 1, g\left(x^{\alpha}\right)$ is called a $\alpha$-order infinite fractional differentiable function defined on $[a, b]$ if $\left({ }_{a} D_{x}^{\alpha}\right)^{n}\left[g\left(x^{\alpha}\right)\right]$ exist for all non-negative integers $n$.

Next, we have the fractional differential operator theorem.

Theorem 3.2: If $0<\alpha \leq 1, z$ is a complex variable, $f(z)=\sum_{n=0}^{\infty} a_{n} z^{n}$, and $g\left(x^{\alpha}\right)$ is a $\alpha$-order infinite fractional differentiable function defined on $[a, b]$. Then the fractional differential operator

$$
f\left({ }_{a} D_{x}^{\alpha}\right)\left[g\left(x^{\alpha}\right)\right]=\sum_{n=0}^{\infty} a_{n}\left({ }_{a} D_{x}^{\alpha}\right)^{n}\left[g\left(x^{\alpha}\right)\right],
$$

if $\sum_{n=0}^{\infty} a_{n}\left({ }_{a} D_{x}^{\alpha}\right)^{n}\left[g\left(x^{\alpha}\right)\right]$ exists.

$$
\text { Proof } \begin{aligned}
f\left({ }_{a} D_{x}^{\alpha}\right)\left[g\left(x^{\alpha}\right)\right] & =\left(\sum_{n=0}^{\infty} a_{n}\left({ }_{a} D_{x}^{\alpha}\right)^{n}\right)\left[g\left(x^{\alpha}\right)\right] \\
& =\lim _{m \rightarrow \infty}\left(\sum_{n=0}^{m} a_{n}\left({ }_{a} D_{x}^{\alpha}\right)^{n}\right)\left[g\left(x^{\alpha}\right)\right] \\
& =\lim _{m \rightarrow \infty} \sum_{n=0}^{m} a_{n}\left({ }_{a} D_{x}^{\alpha}\right)^{n}\left[g\left(x^{\alpha}\right)\right] \\
& =\sum_{n=0}^{\infty} a_{n}\left({ }_{a} D_{x}^{\alpha}\right)^{n}\left[g\left(x^{\alpha}\right)\right] .
\end{aligned}
$$

Remark 3.3: If $\alpha=1$, then Theorem 3.2 becomes the traditional differential calculus case.

At first, we find the fractional derivatives of fractional polynomial function. The following lemma is needed.

Lemma 3.4: Let $0<\alpha \leq 1$, and $n, k$ be non-negative integers. Then

$$
\left({ }_{0} D_{x}^{\alpha}\right)^{n}\left[p_{k}\left(x^{\alpha}\right)\right]=\left\{\begin{array}{c}
p_{k-n}\left(x^{\alpha}\right) \quad \text { if } k \geq n, \\
0 \quad \text { if } k<n .
\end{array}\right.
$$

Proof We know if $n=1$,

$$
\left({ }_{0} D_{x}^{\alpha}\right)^{1}\left[p_{k}\left(x^{\alpha}\right)\right]=\left({ }_{0} D_{x}^{\alpha}\right)^{1}\left[\frac{1}{\Gamma(k \alpha+1)} x^{k \alpha}\right]=\frac{1}{\Gamma((k-1) \alpha+1)} x^{(k-1) \alpha}=p_{k-1}\left(x^{\alpha}\right) .
$$

So, by induction, the desired result is easily obtained.

Theorem 3.5: If the assumptions are the same as Lemma 3.4, then 


$$
\left(\sum_{n=0}^{\infty} a_{n}\left({ }_{0} D_{x}^{\alpha}\right)^{n}\right)\left[p_{k}\left(x^{\alpha}\right)\right]=\sum_{n=0}^{k} a_{n} p_{k-n}\left(x^{\alpha}\right) .
$$

Proof $\left(\sum_{n=0}^{\infty} a_{n}\left({ }_{0} D_{x}^{\alpha}\right)^{n}\right)\left[p_{k}\left(x^{\alpha}\right)\right]=\sum_{n=0}^{\infty} a_{n}\left({ }_{0} D_{x}^{\alpha}\right)^{n}\left[p_{k}\left(x^{\alpha}\right)\right] \quad$ (by Theorem 3.2)

$$
=\sum_{n=0}^{k} a_{n} p_{k-n}\left(x^{\alpha}\right) . \quad \text { (by Lemma 3.4) }
$$

q.e.d.

Example 3.6: $\left(\sin \left({ }_{0} D_{x}^{1 / 3}\right)\right)\left[p_{3}\left(x^{1 / 3}\right)\right]=\left({ }_{0} D_{x}^{1 / 3}-\frac{1}{6}\left({ }_{0} D_{x}^{1 / 3}\right)^{3}+\cdots\right)\left[p_{3}\left(x^{1 / 3}\right)\right]$

$$
\begin{aligned}
& =p_{2}\left(x^{1 / 3}\right)-\frac{1}{6} p_{0}\left(x^{1 / 3}\right) \\
& =\frac{1}{\Gamma\left(\frac{5}{3}\right)} x^{2 / 3}-\frac{1}{6}
\end{aligned}
$$

Next, we study the fractional derivatives of fractional exponential function. We also need a lemma.

Lemma 3.7: Let $0<\alpha \leq 1, n$ be a positive integer, and $\lambda$ be a complex number, then

$$
\left({ }_{0} D_{x}^{\alpha}\right)^{n}\left[E_{\alpha}\left(\lambda x^{\alpha}\right)\right]=\lambda^{n} E_{\alpha}\left(\lambda x^{\alpha}\right)
$$

Proof If $n=1$, then $\left({ }_{0} D_{x}^{\alpha}\right)^{1}\left[E_{\alpha}\left(\lambda x^{\alpha}\right)\right]$

$$
\begin{aligned}
& =\left({ }_{0} D_{x}^{\alpha}\right)^{1}\left[\sum_{k=0}^{\infty} \frac{\lambda^{k} x^{k \alpha}}{\Gamma(k \alpha+1)}\right] \\
& =\sum_{k=0}^{\infty} \frac{\left({ }_{0} D_{x}^{\alpha}\right)^{1}\left[\lambda^{k} x^{k \alpha}\right]}{\Gamma(k \alpha+1)} \\
& =\sum_{k=0}^{\infty} \frac{\lambda^{k}\left({ }_{0} D_{x}^{\alpha}\right)^{1}\left[x^{k \alpha}\right]}{\Gamma(k \alpha+1)} \\
& =\sum_{k=1}^{\infty} \frac{\lambda^{k} x^{(k-1) \alpha}}{\Gamma((k-1) \alpha+1)} \\
& =\lambda \sum_{k=0}^{\infty} \frac{\lambda^{k} x^{k \alpha}}{\Gamma(k \alpha+1)} \\
& =\lambda E_{\alpha}\left(\lambda x^{\alpha}\right) .
\end{aligned}
$$

By induction, the desired result holds.

Theorem 3.8: Suppose that $0<\alpha \leq 1, \lambda$ is a complex number, and $f(z)=\sum_{n=0}^{\infty} a_{n} z^{n}$, then

$$
f\left({ }_{a} D_{x}^{\alpha}\right)\left[E_{\alpha}\left(\lambda x^{\alpha}\right)\right]=f(\lambda) \cdot E_{\alpha}\left(\lambda x^{\alpha}\right),
$$

if $f(\lambda)$ exists.

$$
\begin{aligned}
& f\left({ }_{a} D_{x}^{\alpha}\right)\left[E_{\alpha}\left(\lambda x^{\alpha}\right)\right] \\
= & \left(\sum_{n=0}^{\infty} a_{n}\left({ }_{0} D_{x}^{\alpha}\right)^{n}\right)\left[E_{\alpha}\left(\lambda x^{\alpha}\right)\right]
\end{aligned}
$$




$$
\begin{aligned}
& =\sum_{n=0}^{\infty} a_{n}\left({ }_{0} D_{x}^{\alpha}\right)^{n}\left[E_{\alpha}\left(\lambda x^{\alpha}\right)\right] \quad \text { (by Theorem 3.2) } \\
& =\left(\sum_{n=0}^{\infty} a_{n} \lambda^{n}\right) \cdot E_{\alpha}\left(\lambda x^{\alpha}\right) . \quad(\text { by Lemma 3.7) } \\
& =f(\lambda) \cdot E_{\alpha}\left(\lambda x^{\alpha}\right) .
\end{aligned}
$$

q.e.d.

Corollary 3.9: The case $\alpha=1$ in Theorem 3.8 is

$$
f\left(\frac{d}{d x}\right)[\exp (\lambda x)]=f(\lambda) \cdot \exp (\lambda x)
$$

Example 3.10: $\left(\frac{\exp \left({ }_{0} D_{x}^{1 / 2}\right)}{\cos \left({ }_{0} D_{x}^{1 / 2}\right)}\right)\left[E_{1 / 2}\left(4 x^{1 / 2}\right)\right]=\frac{\exp (4)}{\cos (4)} \cdot E_{1 / 2}\left(4 x^{1 / 2}\right)$,

and

$$
\left(\frac{\exp \left(\frac{d}{d x}\right)}{\cos \left(\frac{d}{d x}\right)}\right)[\exp (4 x)]=\frac{\exp (4)}{\cos (4)} \cdot \exp (4 x) .
$$

We next discuss the fractional derivatives of fractional sine and cosine function. Two lemmas are needed.

Lemma 3.11: Assume that $0<\alpha \leq 1, n$ is a positive integer, and $b$ is a real number, then

$$
\left({ }_{0} D_{x}^{\alpha}\right)\left[\sin _{\alpha}\left(b x^{\alpha}\right)\right]=b \cos _{\alpha}\left(b x^{\alpha}\right),
$$

and

$$
\left({ }_{0} D_{x}^{\alpha}\right)\left[\cos _{\alpha}\left(b x^{\alpha}\right)\right]=-b \sin { }_{\alpha}\left(b x^{\alpha}\right) .
$$

Proof

$$
\begin{aligned}
& \left({ }_{0} D_{x}^{\alpha}\right)\left[\sin _{\alpha}\left(b x^{\alpha}\right)\right] \\
= & \left({ }_{0} D_{x}^{\alpha}\right)\left[\sum_{k=0}^{\infty} \frac{(-1)^{k} b^{2 k+1} x^{(2 k+1) \alpha}}{\Gamma((2 k+1) \alpha+1)}\right] \\
= & b \sum_{k=0}^{\infty} \frac{(-1)^{k} b^{2 k} x^{2 k \alpha}}{\Gamma(2 k \alpha+1)} \\
= & b \cos _{\alpha}\left(b x^{\alpha}\right) .
\end{aligned}
$$

On the other hand,

$$
\begin{aligned}
& \left({ }_{0} D_{x}^{\alpha}\right)\left[\cos _{\alpha}\left(b x^{\alpha}\right)\right] \\
= & \left({ }_{0} D_{x}^{\alpha}\right)\left[\sum_{k=0}^{\infty} \frac{(-1)^{k} b^{2 k} x^{2 k \alpha}}{\Gamma(2 k \alpha+1)}\right] \\
= & b \sum_{k=1}^{\infty} \frac{(-1)^{k} b^{2 k-1} x^{(2 k-1) \alpha}}{\Gamma((2 k-1) \alpha+1)} \\
= & -b \sum_{k=0}^{\infty} \frac{(-1)^{k} b^{2 k+1} x^{(2 k+1) \alpha}}{\Gamma((2 k+1) \alpha+1)} \\
= & -b \sin _{\alpha}\left(b x^{\alpha}\right) .
\end{aligned}
$$$$
\text { q.e.d. }
$$

Lemma 3.12: Suppose that the assumptions are the same as Lemma 3.10, then 


$$
\left({ }_{0} D_{x}^{\alpha}\right)^{n}\left[\sin _{\alpha}\left(b x^{\alpha}\right)\right]=b^{n}\left[\cos \frac{n \pi}{2} \cdot \sin _{\alpha}\left(b x^{\alpha}\right)+\sin \frac{n \pi}{2} \cdot \cos \alpha\left(b x^{\alpha}\right)\right] \text {, }
$$

and

$$
\left({ }_{0} D_{x}^{\alpha}\right)^{n}\left[\cos _{\alpha}\left(b x^{\alpha}\right)\right]=b^{n}\left[\cos \frac{n \pi}{2} \cdot \cos _{\alpha}\left(b x^{\alpha}\right)-\sin \frac{n \pi}{2} \cdot \sin _{\alpha}\left(b x^{\alpha}\right)\right] .
$$

Proof Using Lemma 3.10 and by induction, the desired results are easily obtained.

Remark 3.13: In Lemma 3.12, if $\alpha=1$, we obtain the classical differential cases:

$$
\frac{d^{n}}{d x^{n}} \sin (b x)=b^{n} \sin \left(b x+\frac{n \pi}{2}\right)
$$

and

$$
\frac{d^{n}}{d x^{n}} \cos (b x)=b^{n} \cos \left(b x+\frac{n \pi}{2}\right) .
$$

Theorem 3.14: Let the assumptions be the same as Lemma 3.10, and $f(z)=\sum_{n=0}^{\infty} a_{n} z^{n}$, then

$$
f\left({ }_{0} D_{x}^{\alpha}\right)\left[\sin _{\alpha}\left(b x^{\alpha}\right)\right]=\operatorname{Re}[f(i b)] \cdot \sin _{\alpha}\left(b x^{\alpha}\right)+\operatorname{Im}[f(i b)] \cdot \cos _{\alpha}\left(b x^{\alpha}\right),
$$

and

$$
f\left({ }_{0} D_{x}^{\alpha}\right)\left[\cos _{\alpha}\left(b x^{\alpha}\right)\right]=\operatorname{Re}[f(i b)] \cdot \cos _{\alpha}\left(b x^{\alpha}\right)-\operatorname{Im}[f(i b)] \cdot \sin _{\alpha}\left(b x^{\alpha}\right),
$$

if $f(i b) \neq 0$.

$$
\text { Proof } \begin{aligned}
& f\left({ }_{0} D_{x}^{\alpha}\right)\left[\sin _{\alpha}\left(b x^{\alpha}\right)\right] \\
= & \left(\sum_{n=0}^{\infty} a_{n}\left({ }_{0} D_{x}^{\alpha}\right)^{n}\right)\left[\sin _{\alpha}\left(b x^{\alpha}\right)\right] \\
= & \sum_{n=0}^{\infty} a_{n}\left({ }_{0} D_{x}^{\alpha}\right)^{n}\left[\sin _{\alpha}\left(b x^{\alpha}\right)\right] \\
= & \sum_{n=0}^{\infty} a_{n} \cdot b^{n}\left[\cos \frac{n \pi}{2} \cdot \sin _{\alpha}\left(b x^{\alpha}\right)+\sin \frac{n \pi}{2} \cdot \cos _{\alpha}\left(b x^{\alpha}\right)\right] \quad \text { (by Eq. (26)) } \\
= & \sum_{n=0}^{\infty} a_{n} \cdot b^{n}\left[\operatorname{Re}\left[e^{i \frac{n \pi}{2}}\right] \cdot \sin _{\alpha}\left(b x^{\alpha}\right)+\operatorname{Im}\left[e^{i \frac{n \pi}{2}}\right] \cdot \cos _{\alpha}\left(b x^{\alpha}\right)\right] \\
= & \left(\sum_{n=0}^{\infty} a_{n} \cdot \operatorname{Re}\left[(i b)^{n}\right]\right) \cdot \sin _{\alpha}\left(b x^{\alpha}\right)+\left(\sum_{n=0}^{\infty} a_{n} \cdot \operatorname{Im}\left[(i b)^{n}\right]\right) \cdot \cos _{\alpha}\left(b x^{\alpha}\right) \\
= & \operatorname{Re}\left[\sum_{n=0}^{\infty} a_{n} \cdot(i b)^{n}\right] \cdot \sin _{\alpha}\left(b x^{\alpha}\right)+\operatorname{Im}\left[\sum_{n=0}^{\infty} a_{n} \cdot(i b)^{n}\right] \cdot \cos _{\alpha}\left(b x^{\alpha}\right) \\
= & \operatorname{Re}[f(i b)] \cdot \sin _{\alpha}\left(b x^{\alpha}\right)+\operatorname{Im}[f(i b)] \cdot \cos _{\alpha}\left(b x^{\alpha}\right) .
\end{aligned}
$$

Similarly,

$$
\begin{aligned}
& f\left({ }_{0} D_{x}^{\alpha}\right)\left[\cos _{\alpha}\left(b x^{\alpha}\right)\right] \\
= & \left(\sum_{n=0}^{\infty} a_{n}\left({ }_{0} D_{x}^{\alpha}\right)^{n}\right)\left[\cos _{\alpha}\left(b x^{\alpha}\right)\right] \\
= & \sum_{n=0}^{\infty} a_{n}\left({ }_{0} D_{x}^{\alpha}\right)^{n}\left[\cos _{\alpha}\left(b x^{\alpha}\right)\right]
\end{aligned}
$$




$$
\begin{aligned}
& =\sum_{n=0}^{\infty} a_{n} \cdot b^{n}\left[\cos \frac{n \pi}{2} \cdot \cos _{\alpha}\left(b x^{\alpha}\right)-\sin \frac{n \pi}{2} \cdot \sin _{\alpha}\left(b x^{\alpha}\right)\right] \quad \text { (by Eq. (27)) } \\
& =\left(\sum_{n=0}^{\infty} a_{n} \cdot \operatorname{Re}\left[(i b)^{n}\right]\right) \cdot \cos _{\alpha}\left(b x^{\alpha}\right)-\left(\sum_{n=0}^{\infty} a_{n} \cdot \operatorname{Im}\left[(i b)^{n}\right]\right) \cdot \sin _{\alpha}\left(b x^{\alpha}\right) \\
& =\operatorname{Re}[f(i b)] \cdot \cos _{\alpha}\left(b x^{\alpha}\right)-\operatorname{Im}[f(i b)] \cdot \sin _{\alpha}\left(b x^{\alpha}\right) .
\end{aligned}
$$

Example 3.15: $\left(\frac{1}{2\left({ }_{0} D_{x}^{1 / 4}\right)^{2}-4 \cdot{ }_{0} D_{x}^{1 / 4}+1}\right)\left[\sin _{1 / 4}\left(3 x^{1 / 4}\right)\right]$

$$
\begin{aligned}
& =\operatorname{Re}\left[\frac{1}{2(3 i)^{2}-4 \cdot(3 i)+1}\right] \cdot \sin _{1 / 4}\left(3 x^{1 / 4}\right)+\operatorname{Im}\left[\frac{1}{2(3 i)^{2}-4 \cdot(3 i)+1}\right] \cdot \cos _{1 / 4}\left(3 x^{1 / 4}\right) \quad \text { (by Eq. (30)) } \\
& =\frac{-17}{\mathbf{4 3 3}} \cdot \sin _{1 / 4}\left(3 x^{1 / 4}\right)+\frac{\mathbf{1 2}}{\mathbf{4 3 3}} \cdot \cos _{1 / 4}\left(3 x^{1 / 4}\right)
\end{aligned}
$$

Example 3.16: $\left(\frac{1}{3\left({ }_{0} D_{x}^{1 / 2}\right)^{3}+5\left({ }_{0} D_{x}^{1 / 2}\right)^{2}-7 \cdot{ }_{0} D_{x}^{1 / 2}-4}\right)\left[\cos _{1 / 2}\left(6 x^{1 / 2}\right)\right]$

$$
\begin{aligned}
& =\operatorname{Re}\left[\frac{1}{3(6 i)^{3}+5(6 i)^{2}-7 \cdot(6 i)-4}\right] \cdot \cos _{1 / 2}\left(6 x^{1 / 2}\right)-\operatorname{Im}\left[\frac{1}{3(6 i)^{3}+5(6 i)^{2}-7 \cdot(6 i)-4}\right] \cdot \sin _{1 / 2}\left(6 x^{1 / 2}\right) \\
& =\frac{-\mathbf{1 8 4}}{\mathbf{5 0 9 9 5 6}} \cdot \cos _{1 / 2}\left(6 x^{1 / 2}\right)-\frac{\mathbf{6 9 0}}{\mathbf{5 0 9 9 5 6}} \cdot \sin _{1 / 2}\left(6 x^{1 / 2}\right) .
\end{aligned}
$$

\section{APPLICATIONS}

In the following, we use the fractional differential operator method to find the particular solution of non-homogeneous linear FDE with constant coefficients in a unified form.

Theorem 4.1: Suppose that $0<\alpha \leq 1, n$ is a positive integer, $a_{0} . a_{1}, \cdots, a_{n}$ are real constants, and $a_{n} \neq 0$. The non-homogeneous linear FDE with constant coefficients

$$
\left(a_{n}\left({ }_{0} D_{x}^{\alpha}\right)^{n}+a_{n-1}\left({ }_{0} D_{x}^{\alpha}\right)^{n-1}+\cdots+a_{10} D_{x}^{\alpha}+a_{0}\right)\left[y\left(x^{\alpha}\right)\right]=g\left(x^{\alpha}\right)
$$

has the particular solution

$$
y_{p}\left(x^{\alpha}\right)=\left(\frac{1}{a_{n}\left({ }_{0} D_{x}^{\alpha}\right)^{n}+a_{n-1}\left({ }_{0} D_{x}^{\alpha}\right)^{n-1}+\cdots+a_{1}{ }_{0} D_{x}^{\alpha}+a_{0}}\right)\left[g\left(x^{\alpha}\right)\right]
$$

Proof Since

$$
\left(a_{n}\left({ }_{0} D_{x}^{\alpha}\right)^{n}+a_{n-1}\left({ }_{0} D_{x}^{\alpha}\right)^{n-1}+\cdots+a_{10} D_{x}^{\alpha}+a_{0}\right)\left[\left(\frac{1}{a_{n}\left({ }_{0} D_{x}^{\alpha}\right)^{n}+a_{n-1}\left({ }_{0} D_{x}^{\alpha}\right)^{n-1}+\cdots+a_{10} D_{x}^{\alpha}+a_{0}}\right)\left[g\left(x^{\alpha}\right)\right]\right]=
$$

$g\left(x^{\alpha}\right)$

the desired result holds.

q.e.d.

The case $\alpha=1$ in Theorem 4.1 is the traditional non-homogeneous linear ordinary differential equation with constant coefficients result.

Corollary 4.2: The non-homogeneous linear ordinary differential equation with constant coefficients 


$$
\left(a_{n} \frac{d^{n}}{d x^{n}}+a_{n-1} \frac{d^{n-1}}{d x^{n-1}}+\cdots+a_{1} \frac{d}{d x}+a_{0}\right)[y(x)]=g(x)
$$

has the particular solution

$$
y_{p}(x)=\left(\frac{1}{a_{n} \frac{d^{n}}{d x^{n}}+a_{n-1} \frac{d^{n-1}}{d x^{n-1}+\cdots+a_{1} \frac{d}{d x}+a_{0}}}\right)[g(x)] .
$$

Next, we study Eq. (34) for the cases that $g\left(x^{\alpha}\right)$ are several special fractional functions.

Theorem 4.3: If the assumptions are the same as Theorem 4.1, and $A, B, C, D, b$ are real numbers. Let $f(z)=$ $\frac{1}{a_{n} z^{n}+a_{n-1} z^{n-1}+\cdots+a_{1} z+a_{0}}=\sum_{m=0}^{\infty} c_{m} z^{m}$. Consider the non-homogeneous linear FDE with constant coefficients

$$
\left(a_{n}\left({ }_{0} D_{x}^{\alpha}\right)^{n}+a_{n-1}\left({ }_{0} D_{x}^{\alpha}\right)^{n-1}+\cdots+a_{10} D_{x}^{\alpha}+a_{0}\right)\left[y\left(x^{\alpha}\right)\right]=g\left(x^{\alpha}\right) .
$$

Case 1. If $g\left(x^{\alpha}\right)=A \cdot p_{k}\left(x^{\alpha}\right)$, then Eq. (38) has the particular solution

$$
y_{p}\left(x^{\alpha}\right)=A \cdot \sum_{m=0}^{k} c_{m} p_{k-m}\left(x^{\alpha}\right) .
$$

Case 2. If $g\left(x^{\alpha}\right)=B \cdot E_{\alpha}\left(b x^{\alpha}\right)$, then

$$
y_{p}\left(x^{\alpha}\right)=\frac{B}{a_{n} b^{n}+a_{n-1} b^{n-1}+\cdots+a_{1} b+a_{0}} \cdot E_{\alpha}\left(b x^{\alpha}\right),
$$

if $a_{n} b^{n}+a_{n-1} b^{n-1}+\cdots+a_{1} b+a_{0} \neq 0$.

Case 3. If $g\left(x^{\alpha}\right)=C \cdot \sin _{\alpha}\left(b x^{\alpha}\right)$, we obtain

$$
y_{p}\left(x^{\alpha}\right)=C \cdot\left\{\operatorname{Re}[f(i b)] \cdot \sin _{\alpha}\left(b x^{\alpha}\right)+\operatorname{Im}[f(i b)] \cdot \cos _{\alpha}\left(b x^{\alpha}\right)\right\},
$$

if $a_{n}(i b)^{n}+a_{n-1}(i b)^{n-1}+\cdots+a_{1}(i b)+a_{0} \neq 0$

Case 4. If $g\left(x^{\alpha}\right)=D \cdot \cos _{\alpha}\left(b x^{\alpha}\right)$, then

$$
y_{p}\left(x^{\alpha}\right)=D \cdot\left\{\operatorname{Re}[f(i b)] \cdot \cos _{\alpha}\left(b x^{\alpha}\right)-\operatorname{Im}[f(i b)] \cdot \sin _{\alpha}\left(b x^{\alpha}\right)\right\},
$$

if $a_{n}(i b)^{n}+a_{n-1}(i b)^{n-1}+\cdots+a_{1}(i b)+a_{0} \neq 0$.

Proof By Theorem 3.5, Theorem 3.8, and Theorem 3.14, the Case 1-4 hold.

q.e.d.

The case $\alpha=1$ is in Theorem 4.3 is the result of the non-homogeneous linear ordinary differential equation with constant coefficients.

Corollary 4.4: If the assumptions are the same as Theorem 4.3. Consider the non-homogeneous linear ordinary differential equation with constant coefficients

$$
\left(a_{n} \frac{d^{n}}{d x^{n}}+a_{n-1} \frac{d^{n-1}}{d x^{n-1}}+\cdots+a_{1} \frac{d}{d x}+a_{0}\right)[y(x)]=g(x) .
$$

Case 1. If $g(x)=A \cdot p_{k}(x)=\frac{A}{k !} x^{k}$, then the particular solution of Eq. (43) is 


$$
y_{p}(x)=A \cdot \sum_{m=0}^{k} c_{m} p_{k-m}(x) .
$$

Case 2. If $g(x)=B \cdot \exp (b x)$, we have

$$
y_{p}(x)=\frac{B}{a_{n} b^{n}+a_{n-1} b^{n-1}+\cdots+a_{1} b+a_{0}} \exp (b x),
$$

if $a_{n} b^{n}+a_{n-1} b^{n-1}+\cdots+a_{1} b+a_{0} \neq 0$.

Case 3. If $g(x)=C \cdot \sin (b x)$, then

$$
y_{p}(x)=C \cdot\{\operatorname{Re}[f(i b)] \cdot \sin (b x)+\operatorname{Im}[f(i b)] \cdot \cos (b x)\},
$$

if $a_{n}(i b)^{n}+a_{n-1}(i b)^{n-1}+\cdots+a_{1}(i b)+a_{0} \neq 0$.

Case 4. If $g(x)=D \cdot \cos (b x)$, then

$$
y_{p}(x)=D \cdot\{\operatorname{Re}[f(i b)] \cdot \cos (b x)-\operatorname{Im}[f(i b)] \cdot \sin (b x)\},
$$

if $a_{n}(i b)^{n}+a_{n-1}(i b)^{n-1}+\cdots+a_{1}(i b)+a_{0} \neq 0$.

Next, we give some examples to illustrate our results.

Example 4.5: The particular solution of the non-homogeneous linear FDE with constant coefficients

$$
\left(5\left({ }_{0} D_{x}^{1 / 2}\right)^{3}-2\left({ }_{0} D_{x}^{1 / 2}\right)^{2}-4{ }_{0} D_{x}^{1 / 2}+3\right)\left[y\left(x^{1 / 2}\right)\right]=6 \cdot p_{2}\left(x^{1 / 2}\right)
$$

is

$$
\begin{aligned}
& y_{p}\left(x^{1 / 2}\right)=\left(\frac{1}{5\left({ }_{0} D_{x}^{1 / 2}\right)^{3}-2\left({ }_{0} D_{x}^{1 / 2}\right)^{2}-4{ }_{0} D_{x}^{1 / 2}+3}\right)\left[6 \cdot p_{2}\left(x^{1 / 2}\right)\right] \\
& =6\left(\frac{1}{3}+\frac{4}{9}{ }_{0} D_{x}^{1 / 2}+\frac{22}{27}\left({ }_{0} D_{x}^{1 / 2}\right)^{2}+\cdots\right)\left[p_{2}\left(x^{1 / 2}\right)\right] \\
& =6\left(\frac{1}{3} p_{2}\left(x^{1 / 2}\right)+\frac{4}{9} p_{1}\left(x^{1 / 2}\right)+\frac{22}{27} p_{0}\left(x^{1 / 2}\right)\right) \\
& =2 \cdot p_{2}\left(x^{1 / 2}\right)+\frac{8}{3} \cdot p_{1}\left(x^{1 / 2}\right)+\frac{44}{9} \cdot p_{0}\left(x^{1 / 2}\right) \\
& =2 x+\frac{16}{3 \sqrt{\pi}} \cdot x^{1 / 2}+\frac{44}{9} \text {. }
\end{aligned}
$$

\section{Example 4.6:}

$$
\left(\left({ }_{0} D_{x}^{1 / 3}\right)^{2}+2{ }_{0} D_{x}^{1 / 3}+3\right)\left[y\left(x^{1 / 3}\right)\right]=4 \cdot E_{1 / 3}\left(2 x^{1 / 3}\right)
$$

has the particular solution

$$
y_{p}\left(x^{1 / 3}\right)=\frac{4}{11} \cdot E_{1 / 3}\left(2 x^{1 / 3}\right)
$$


Example 4.7: The particular solution of

$$
\left(2\left({ }_{0} D_{x}^{1 / 4}\right)^{2}-4 \cdot{ }_{0} D_{x}^{1 / 4}+1\right)\left[y\left(x^{1 / 4}\right)\right]=\sin _{1 / 4}\left(3 x^{1 / 4}\right)
$$

is

$$
\begin{aligned}
y_{p}\left(x^{1 / 3}\right) & =\left(\frac{1}{2\left({ }_{0} D_{x}^{1 / 4}\right)^{2}-4 \cdot{ }_{0} D_{x}^{1 / 4}+1}\right)\left[\sin _{1 / 4}\left(3 x^{1 / 4}\right)\right] \\
& =\frac{-17}{433} \cdot \sin _{1 / 4}\left(3 x^{1 / 4}\right)+\frac{12}{433} \cdot \cos _{1 / 4}\left(3 x^{1 / 4}\right) . \quad \text { (by Eq. (32)) }
\end{aligned}
$$

\section{Example 4.7:}

$$
\left(3\left({ }_{0} D_{x}^{1 / 2}\right)^{3}+5\left({ }_{0} D_{x}^{1 / 2}\right)^{2}-7 \cdot{ }_{0} D_{x}^{1 / 2}-4\right)\left[y\left(x^{1 / 2}\right)\right]=\cos _{1 / 2}\left(6 x^{1 / 2}\right)
$$

has the particular solution

$$
\begin{aligned}
y_{p}\left(x^{1 / 2}\right) & =\left(\frac{1}{3\left({ }_{0} D_{x}^{1 / 2}\right)^{3}+5\left({ }_{0} D_{x}^{1 / 2}\right)^{2}-7 \cdot{ }_{0} D_{x}^{1 / 2}-4}\right)\left[\cos _{1 / 2}\left(6 x^{1 / 2}\right)\right] \\
& =\frac{-\mathbf{1 8 4}}{\mathbf{5 0 9 9 5 6}} \cdot \cos _{1 / 2}\left(6 x^{1 / 2}\right)-\frac{\mathbf{6 9 0}}{\mathbf{5 0 9 9 5 6}} \cdot \sin _{1 / 2}\left(6 x^{1 / 2}\right)
\end{aligned}
$$

\section{CONCLUSION}

There are several methods to find the particular solution of fractional differential equations, and the solution depends on the type of fractional derivative used. In the present paper, the fractional differential operator method are used to obtain the particular solution of non-homogeneous linear FDE with constant coefficients, depends on Jumarie type of modified R-L fractional derivative. Some examples proposed in this article concerned with the fractional derivatives of some fractional functions, and their applications to demonstrate the validity of our results. At the same time, our method is the generalization of the one to obtain the particular solution of classical non-homogeneous linear differential equations with constant coefficients.

\section{REFERENCES}

[1] I. Podlubny, Fractional differential equations, Mathematics in Science and Engineering, Academic Press, San Diego, California, USA. 1999; 198.

[2] S. Das, Functional fractional calculus, 2nd Edition, Springer-Verlag, 2011.

[3] E. Ahmed, El-Sayed AMA, and El-Saka HAA, “Equilibrium points, stability and numerical solutions of fractional-order predator-prey and rabies models", Journal of Mathematical Analysis and Applications, 2007:325, p. $542-53$.

[4] A. Alsaedi, S. K Ntouyas, R. P Agarwal, and B. Ahmad, "On Caputo type sequential fractional differential equations with nonlocal integral boundary conditions", Advances in Difference Equations 2015:33, p. 1-12.

[5] K. S. Miller, Ross B., An introduction to the fractional calculus and fractional differential equations, John Wiley \& Sons, New York, NY, USA, 1993. 
[6] S. S. Ray, R.K. Bera, “An approximate solution of a nonlinear fractional differential equation by Adomian decomposition method ", Applied Mathematics and Computation, 2005:167, p. 561-571.

[7] K. Diethelm, The analysis of Fractional Differential equations. Springer-Verlag, 2010.

[8] D. Kumar and J. Daiya, "Linear fractional non-homogeneous differential equations with Jumarie fractional derivative", Journal of Chemical, Biological and Physical Sciences, 2016, 6(2), p. 607-618.

[9] U. Ghosh, S. Sengupta, S. Sarkar and S. Das, “ Analytic solution of linear fractional differential equation with Jumarie derivative in term of Mittag-Leffler function", American Journal of Mathematical Analysis, 2015, 3(2), p. 32-38.

[10] S. L. Khalaf, "Particular solution of linear fractional differential equation with constant coefficients by inverse operators", International Journal of Pure and Applied Research in Engineering and Technology, 2016, 4(7), p. 48-63.

[11] S. L. Khalaf, "Analytic solution of linear fractional differential equations with constant coefficients", Mathematical Theory and Modeling, 2016, 6(2), p. 135-154.

[12] J. C. Prajapati, "Certain properties of Mittag-Leffler function with argument $x^{\alpha}, \alpha>0$ ", Italian Journal of Pure and Applied Mathematics, 2013, 30, p. 411-416.

[13] K. V. Zhukovsky, "Inverse derivative and solutions of some ordinary differential equations", Applied Mathematics, 2012, 2(2), p. 34-39. 